\title{
Modelos cognitivos formales: Una herramienta para la integración del conocimiento en psicología cognitiva y neurociencia cognitiva
}

\author{
Formal cognitive models: A tool for the knowledge integration \\ of cognitive psychology and cognitive neuroscience
}

\author{
Odir Antonio Rodríguez-Villagra ${ }^{1}$ \\ Universidad de Costa Rica, Costa Rica
}

\begin{abstract}
Resumen. El objetivo de la presente revisión fue proponer algunas recomendaciones para aquellos psicólogos cognitivos interesados en incorporar el estudio del sistema nervioso en sus investigaciones. Primero, son presentadas algunas resistencias de científicos cognitivos y neurocientíficos para incorporar las teorías de la neurociencia o la psicología cognitiva en sus respectivas áreas de estudio. Luego, se exponen algunos riesgos relacionados con el atractivo de las neurociencias. Segundo, se proponen los modelos cognitivos formales como una herramienta importante en la integración del conocimiento entre la psicología cognitiva y la neurociencia cognitiva. Tercero, se presentan ejemplos de cómo los modelos cognitivos formales ayudan al razonamiento en el contexto de la investigación en neurociencia cognitiva. Finalmente, se enumeran algunas recomendaciones con el afán de promover un inicio promisorio en la investigación en neurociencia cognitiva.
\end{abstract}

Palabras clave. Psicología cognitiva, neurociencia cognitiva, modelos cognitivos formales y razonamiento.

Abstract. The aim of this review was to propose some pieces of advice to cognitive psychologists interested in incorporate the study of the nervous system on their researches. First, this work presents the scholars' resistances to take into account theories and findings from neurosciences or cognitive psychology in their respective research area; then, some risks related to the allure of neurosciences are offered. Second, formal cognitive models are proposed as an important tool to incorporate in the cognitive neuroscience research. Third, some arguments and examples showing how formal cognitive models aid reasoning and research in cognitive neuroscience are given. Finally, some proposals are presented in order to promote a suitable start in the cognitive neuroscience research.

Key words. Cognitive psychology, Cognitive neuroscience, formal cognitive models and reasoning.

\footnotetext{
${ }^{1}$ Odir Antonio Rodríguez-Villagra, Centro de Investigación en Neurociencias e Instituto de Investigaciones Psicológicas, Universidad de Costa Rica. Direccion postal: Vicerrectoría de Investigación, 1501-2060. Ciudad Universitaria “Rodrigo Facio", Universidad de Costa Rica. San Pedro de Montes de Oca. San José, Costa Rica. E-mail: odir.rodriguez@ucr.ac.cr
}

\section{(@) $\odot \odot$}

Esta obra está bajo una Licencia Creative Commons Atribución-NoComercial-SinDerivar 4.0 Internacional. 


\section{Introducción}

El estudio y la comprensión de la cognición humana ha tenido un progreso considerable gracias al trabajo multidisciplinario de psicólogos cognitivos y neurocientíficos de la cognición humana (Corrado \& Doya, 2007; Dolan, 2008; O’Doherty, Hampton \& Kim, 2007).

El desarrollo natural del conocimiento, la necesidad de abordar ciertos problemas de investigación de manera multidisciplinaria, la apasionante idea de empezar a entender los fenómenos mentales en función del sistema nervioso, entre otros aspectos, hace que los psicólogos cognitivos se vean tentados o en la necesidad de aventurase en el mundo de las neurociencias cognitivas. El objetivo del presente trabajo es señalar algunos aspectos que sirvan de reflexión para quienes desean incorporar a sus líneas de investigación un enfoque neurocientífico.

En esta sección se ofrece una breve descripción de la psicología cognitiva y la neurociencia cognitiva con el propósito de resaltar similitudes y diferencias entre estas áreas del conocimiento; lo que da pie a la presentación de algunas limitaciones que pueden encontrar los científicos cognitivos a la hora de incorporar un enfoque neurocientífico en sus líneas de investigación. Luego en secciones subsiguientes se presentan: a) algunas resistencias y riesgos respecto a la integración del conocimiento procedente de la psicología cognitiva y las neurociencias afines; b) se señala el papel de los modelos cognitivos formales (i.e., modelos formales) como herramienta fundamental para el avance de la neurociencia cognitiva; c) se exponen algunos ejemplos de cómo los modelos formales pueden contribuir con el desarrollo de la neurociencia cognitiva y; d) se presentan algunas recomendaciones para aquellas personas interesadas en aventurarse en el mundo de las neurociencias cognitivas.

La psicología cognitiva es el estudio de la representación del conocimiento, lo que involucra entender como las personas perciben, aprenden, recuerdan, piensan y sienten acerca de la información (Sternberg \& Sternberg, 2012). Por su parte, la neurociencia cognitiva estudia la forma en que el sistema nervioso implementa diferentes procesos cognitivos (Forstmann, Wagenmakers, Eichele, Brown \& Serences, 2011) mediante la examinación de la actividad neuronal involucrada con los procesos de interés.

El eje fundamental para la evaluación de hipótesis y desarrollo de teorías en ambas áreas de conocimiento es el diseño experimental, no obstante, estrategias como los auto reportes, los estudios de caso, las observaciones de situaciones de la "vida real" (e.g., estudios clínicos), los modelos formales (i.e., modelos matemáticos o computacionales), entre otras, han sido una fuente invaluable de apoyo para las teorías acerca de la cognición humana. Además, la neurociencia cognitiva centra su análisis en datos generados por métodos como: el registro de la actividad eléctrica cerebral mediante electroencefalogramas (EEGs; Sauseng, et al., 2009); cambios en la actividad eléctrica cerebral debido a un evento o los comúnmente llamados potenciales evocados relacionados con eventos (ERP; Vogel, MacCollough, \& Machizawa, 2005); el registro de la actividad metabólica cerebral por medio de la Tomografía por Emisión de Positrones (PET; Kim et al., 2002), imágenes de resonancia magnética funcional (fMRI, Naughtin, Mattingley \& Dux, 2014), la estimulación magnética transcraneal (TMS; Klimesch, et al. 2009), entre otros.

La correspondencia entre las áreas de conocimiento mencionadas, a saber, un objeto de estudio en común pero dirigido a distintos niveles de descripción (i.e., nivel cognitivo-conductual y el nivel neural) y metodologías de análisis similares, hace prever que la neurociencia cognitiva y la psicología cognitiva deberían integrar sus respectivas teorías con cierta naturalidad; empero, este no parece ser el caso. En la siguiente sección se sugieren algunos aspectos que han dificultado la integración del conocimiento acumulado proveniente de la psicología cognitiva y las neurociencias afines.

\section{Resistencias y riesgos a la integración del conocimiento}

Resistencias. Sin duda la integración racional y parsimoniosa de teorías y metodologías de diversas áreas de conocimiento es necesaria para el estudio de la cognición humana. Sin embargo, tanto en la psicología cognitiva y en las neurociencias existen posiciones divergentes respecto 
al papel de estas áreas de conocimiento en el estudio de la cognición humana. Una primera posición sostiene que las teorías desarrolladas en el ámbito de la psicología cognitiva son fundamentales para el desarrollo de la neurociencia cognitiva; específicamente, Colthear (2012) plantea que la psicología cognitiva y la neurociencia cognitiva responden a niveles distintos de descripción. El primero de ellos responde al nivel cognitivo-conductual del estudio de la cognición humana, mientras que el segundo alude al nivel neuronal de la cognición humana. Según Colthear el nivel cognitivo tiene un estatus e importancia superior con respecto al nivel neuronal del estudio de la cognición. Entre otros aspectos, la posición de Colthear se basa en el enfoque de Kihlstrom (2010), el cual es descrito en la siguiente cita "La psicología sin neurociencia sigue siendo aún una ciencia de la vida mental, pero la neurociencia sin psicología no es más que la ciencia de las neuronas" (p. 762). El razonamiento detrás de esta cita sugiere que cualquier interés en teorizar acerca de la cognición humana al nivel neural debe ser guiado por teorías especificadas a nivel cognitivo (Colthear, 2012; Gallistel, 1999).

Una segunda posición respecto a los aportes de la psicología cognitiva hacia la neurociencia sostiene que éstos son pocos y se caracterizan por ser confusos, incoherentes e incluso risibles (Bennett \& Hacker, 2006). Dichos autores fundamentan su posición en un análisis histórico y crítico del estudio de las habilidades lingüísticas desde los estudios de Broca y Wernicke hasta los estudios cognitivos que involucran técnicas de imagen no invasivas.

Una de las críticas más importantes se refiere a la falacia mereológica de la neurociencia la cuál es descrita como adscribir al cerebro -una parte del agente- atributos que sólo pueden ser adscritos al agente como un todo. Es decir, el cerebro humano no piensa, somos los seres humanos como un todo los que pensamos. Dicha falacia no desacredita la gran mayoría de hallazgos encontrados a la fecha, después de todo, ningún científico creerá que es literalmente el cerebro quien piensa.

Además, Bennett y Hacker (2006) argumentan que las teorías cognitivas son meras descripciones de las habilidades lingüísticas y por lo tanto carecen de cualquier poder explicativo. En este aspecto y contrario a la propuesta de Benneth y Hacker, Coltheart (2012) presenta argumentos y evidencia de que las teorías cognitivas de las habilidades lingüísticas no son únicamente descripciones del fenómeno en cuestión. Desafortunadamente la propuesta de Bennett y Hacker (2006) carece de alternativas para solventar los problemas teóricos y metodológicos que la psicología cognitiva y ciencias afines han arrastrado a través del tiempo. Lo que hace que la propuesta de estos científicos sea poco fructífera.

En mi opinión la primera posición (Coltheart, 2012; Gallistel, 1999; Kihlstrom, 2012) deja una muy buena enseñanza: las teorías al nivel cognitivo deben guiar mas no limitar la investigación en neurociencia cognitiva. Lo radical de la segunda propuesta (Bennett \& Hacker, 2006) hace que su enseñanza deba ser tomada con mucha cautela.

A modo de resumen, es claro que posiciones radicales como la de Bennett y Hacker (2006) dificultan la integración de teorías desarrolladas desde diferentes campos de conocimiento. Por otra parte, posiciones constructivas y críticas (Brown, 2012; Coltheart, 2012; Forstmann, et al. 2011; Gallistel, 1999; Kihlstrom, 2012) respecto a la relación entre los dos niveles de descripción de la cognición humana (i.e., cognitivo y neural) facilitarán el desarrollo de la neurociencia cognitiva.

Riesgos. La psicología cognitiva y neurociencia cognitiva pueden abordar fenómenos desde teorías distintas y no necesariamente complementarias o compatibles. Lewandowsky, Ecker, Farrell y Brown (2012) presentan un ejemplo de ello; en la literatura neurocientífica existe consenso acerca de que una vez que las memorias son codificadas se inicia un proceso de consolidación (Wixted, 2004). La consolidación se refiere al proceso por el cual las memorias son estabilizadas (i.e., fortalecidas) con el transcurrir del tiempo (Lewandowsky, et al., 2012). Concretamente, varios estudios sugieren que el sueño y la estimulación magnética transcraneal favorecen el almacenamiento a largo plazo de ciertos tipos de información (Peigneux, Schmitz \& Urbain, 2010; Wixted, 2010). 
La consolidación como constructo en la psicología cognitiva es raramente tomada en cuenta en los modelos memoria, de hecho, estos modelos ofrecen explicaciones satisfactorias sin aludir al constructo en cuestión (Brown \& Lewandowsky, 2010; Oberauer \& Kliegl, 2006; Oberauer, Lewandowsky, Farrell, Jarrod \& Greaves, 2012). Esta disyuntiva entre las teorías cognitivas y las teorías en neurociencia no descalifica la utilidad de la consolidación como constructo pero sugiere que el fenómeno de la consolidación y las teorías cognitivas deben ser reexaminadas.

Otro riesgo asociado con la integración del conocimiento entre la psicología cognitiva y la neurociencia cognitiva surge del atractivo inherente de las neurociencias (Weisberg, Keil, Goodstein, Rawson \& Gray, 2008). En esta línea Weisberg et al. (2008) desarrollaron un estudio en el que estudiantes universitarios sin educación formal en neurociencias (i.e., novatos), estudiantes de un curso de neurociencia cognitiva y expertos en el tema juzgaron explicaciones concernientes a un fenómeno en particular. En un diseño factorial cruzaron dos factores: tipo de explicación (i.e., buena vs mala) y naturaleza de la explicación (i.e., con componentes neurocientíficos vs sin componentes neurocientíficos). El experimento demostró que el grupo de novatos y los estudiantes de neurociencia cognitiva calificaron más satisfactoriamente las explicaciones con componentes neurocientíficos que las explicaciones que no lo incluían; además, las malas explicaciones fueron mejor valoradas si el componente neurocientífico estaba presente. El grupo de expertos en neurociencias no presentó sesgo relacionado con los contenidos neurocientíficos.

En el trabajo de Weisberg et al. (2008) las malas explicaciones eran reformulaciones circulares del fenómeno, por lo tanto, no eran explicativas. Aunque el estudio anterior refleja y apoya la creencia de que en ciertas circunstancias las personas tienen problemas evaluando explicaciones (Evans, 2002; Weisberg et al. 2008; Tversky \& Kahneman, 1983) es importante señalar que en contextos argumentativos las personas tienden a mostrar mayores destrezas evaluando y produciendo argumentos (Mercier \& Sperber, 2011).
Entendido así, es plausible proponer que el sesgo asociado con el atractivo de las neurociencias debe ser menor en el contexto científico ya que es un contexto claramente argumentativo. El hecho de que en la investigación de Weisberg et al. (2008) los neurocientíficos no mostraran un sesgo al evaluar explicaciones sustenta, en cierta medida, lo propuesto en la oración anterior.

En la sección siguiente se propone que el razonamiento, entendido como la consciencia de que la relación entre los argumentos y las premisas justifica la conclusión (Mercier \& Sperber, 2011), puede ser potenciado mediante la implementación de modelos formales de la cognición humana.

\section{Modelos cognitivos formales}

Las primeras fases de un proyecto de investigación inician con una pregunta de investigación ambigua; después de una revisión detallada de la literatura la pregunta de investigación puede ser formulada de manera precisa permitiendo establecer hipótesis dentro de una teoría consolidada. En psicología cognitiva, así como en la neurociencia cognitiva, la mayoría de las teorías han sido desarrolladas verbalmente, a saber, descripciones de sus premisas empleando el lenguaje natural. Las teorías verbales son importantes para resumir y organizar un conjunto común de regularidades empíricas acumuladas a partir de la investigación. A pesar de su importancia, se ha señalado que las teorías verbales no especifican ciertos detalles con implicaciones teóricas importantes (Farrell \& Lewandowsky, 2010; Lewandowsky \& Farrell, 2011; Lewandowsky, 1993).

Lo anterior no se debe al descuido de los científicos, más bien, es producto de la ambigüedad de los presupuestos de las teorías en psicología cognitiva y en neurociencia cognitiva. En cualquier teoría verbal hay muchos aspectos que no han sido especificados. A continuación se presenta un ejemplo en el contexto del estudio de la memoria de trabajo.

La Figura 1 ilustra una tarea típica de memoria de trabajo (ver Tarea A); en este tipo de tareas los participantes son instruidos para que codifiquen (i.e., 
memorizar) una serie de letras que son presentadas secuencialmente. Entre la codificación de cada una de las letras se presentan operaciones matemáticas simples (e.g., multiplicaciones) que deben ser juzgadas ya sea como "verdaderas" o "falsas" (ver Figura 1).

Después de que los participantes han codificado varias letras y juzgado las operaciones deben recuperar (e.g., decir en vOz alta) las letras en el orden presentado. La Figura 1 esquematiza el tiempo que consumen las diferentes fases de la tarea: el sombreado negro representa el tiempo dedicado a la codificación (i.e., fase de codificación); el sombreado gris representa el tiempo dedicado al procesamiento de la información, esto es, el tiempo empleado en el juzgamiento de la operación matemática (i.e., fase de procesamiento) y; el tiempo libre entre las fases de procesamiento y codificación es denotado por las partes en blanco. Como ilustra la figura las Tareas A y B difieren únicamente en la cantidad de operaciones matemáticas a juzgar. Consecuentemente, los participantes tienen más tiempo libre durante la Tarea B.

El modelo de recursos compartidos basado en el tiempo (TBRS, por sus siglas en inglés, Barrouillet, Bernardin \& Camos, 2004; Barrouillet, De Paepe \& Langerock, 2012) predice que el rendimiento de las personas en la Tarea A debería ser inferior al rendimiento de las personas en la Tarea B. El modelo TBRS propone que el mantenimiento y el procesamiento de

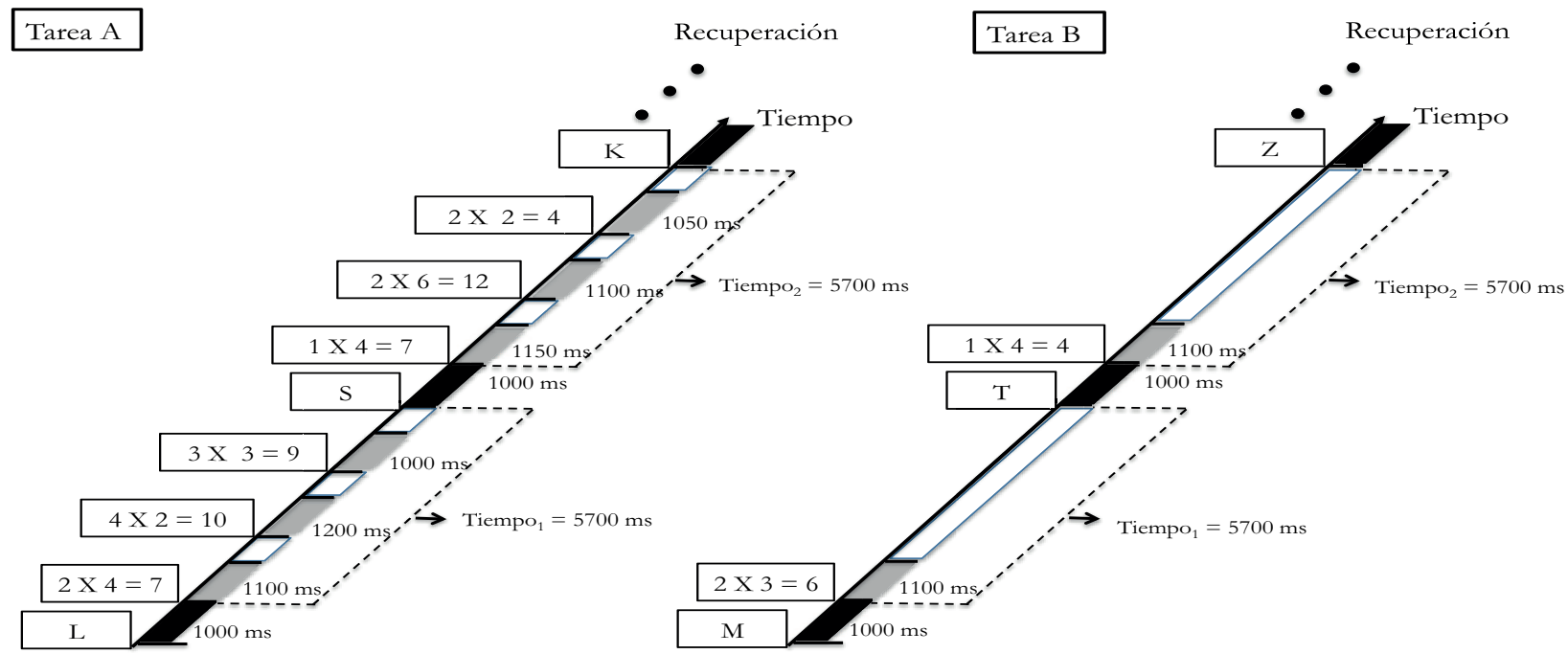

$$
\begin{aligned}
& \text { Parámetros para la tarea A } \\
& t_{a}=1100+1200+1000+1150+1100+1050 \\
& t_{a}=6600 \\
& \text { Tiempo Total }(T)=\text { Tiempo }_{1}+\text { Tiempo }_{2} \\
& T=11400 \mathrm{~ms} \\
& C L=6600 / 11400 \\
& C L=0.57
\end{aligned}
$$

$$
\begin{aligned}
& \text { Parámetros para la tarea B } \\
& t_{a}=1100+1100 \\
& t_{a}=2200 \\
& \text { Tiempo Total }(T)=\text { Tiempo }_{1}+\text { Tiempo }_{2} \\
& T=11400 \mathrm{~ms} \\
& C L=2200 / 11400 \\
& C L=0.19
\end{aligned}
$$

Figura 1. Representación esquemática de una tarea para evaluar memoria de trabajo. La Tarea A y la Tarea B representan diferentes manipulaciones experimentales para ejemplificar la estimación de la carga cognitiva (CL, Barrouillet, Bernardin y Camos, 2004; Barrouillet, De Paepe y Langerock, 2012). Los recuadros en la parte inferior de la figura detallan la explicación de la estimación de la CL. 
la información comparten el mismo recurso atencional, por lo que no es posible mantener y procesar de manera simultánea un conjunto de representaciones.

Este modelo asume que mientras el recurso atencional es dedicado al procesamiento de la información, las representaciones codificadas sufren decaimiento relacionado con el transcurso del tiempo. Este decaimiento puede ser restaurado vía ensayo sub articulatorio -esto es la recitación mental de la memoranda (Baddeley, Thomson, \& Buchanan, 1975; Kieras, Meyer, Mueller \& Seymour, 1999; Page \& Norris, 1998) -o ensayo vía atención- esto es redirigir la atención a la memoranda (Camos, Lagner $\&$ Barrouillet, 2009). Una predicción que se desprende del modelo TBRS es que la probabilidad de recuperar exitosamente la memoranda es función de la carga cognitiva ( $C L$, por sus siglas en inglés) la cual es dada por la siguiente ecuación:

$$
C L=t_{a} / T
$$

Aquí, $t_{a}$ es el tiempo dedicado al procesamiento de la información y $\mathrm{T}$ es el tiempo total disponible para procesar la información. Siguiendo la ecuación la $C L$ estimada para la Tarea A es igual a $0.57=6600$ $\mathrm{ms} / 11400 \mathrm{~ms}$, en donde 6600 corresponde al estimado de $t_{a}$ y 11400 corresponde $T$ (para detalles ver recuadro inferior izquierdo de la Figura 1). La CL para la Tarea $\mathrm{B}$ es igual a 0.19 (para detalles ver recuadro inferior izquierdo de la Figura 1). De acuerdo con el estimado de la $C L$ el modelo TBRS predice que es más difícil recuperar la memoranda en la Tarea A que en la Tarea B. Lo anterior se debe a que en la Tarea B, comparada con la Tarea A, existe una mayor proporción de tiempo libre lo cual permite que este tiempo pueda ser empleado en la restauración de la memoranda ya sea vía ensayo sub articulatorio o ensayo vía atención. Consecuentemente, la mayor activación de las representaciones en el contexto de la Tarea B facilitaría su recuperación al final del ensayo.

A pesar de que un buen número de trabajos apoya las predicciones de esta teoría verbal (Barrouillet, Bernardin \& Camos, 2004; Barrouillet, De Paepe \& Langerock, 2012) el presupuesto del decaimiento de las trazas en memoria debido al transcurrir del tiempo no especifica si el decaimiento de su activación sigue una función lineal o exponencial; además, la teoría no indica la rapidez y el orden en el cual las representaciones en memoria son reactivadas, no especifica la naturaleza de las representaciones (e.g., localizaciones, distribuidas), entre otros. Clarificar estas interrogantes es necesario para llevar esta teoría un paso más adelante en su desarrollo. Dichos aspectos sólo pueden ser contrastados si se especifican en el contexto de los modelos formales.

A pesar de estas limitaciones, existe la posibilidad de implementar las teorías verbales en modelos cognitivos formales. Las teorías instanciadas como modelos formales pueden ser especificadas detalladamente, resultando en una teoría internamente consistente. Los modelos formales son descritos en matemática formal o programas de computadora y son derivados a partir de los principios básicos de la cognición.

Un aspecto distintivo de los modelos cognitivos formales es que los investigadores pueden usarlos para hacer predicciones cuantitativas bastante precisas. Oberauer y Lewandowsky (2011) reprodujeron predicciones básicas del modelo TBRS; entre otras cosas, estos autores predijeron el nivel de activación de las trazas en memoria en un momento dado como función del decaimiento debido al transcurrir del tiempo y como función del ensayo vía atención (Oberauer \& Lewandowsky, 2011). Más importante aún, este modelo fue capaz de ajustar varios conjuntos de datos que la teoría verbal previamente había predicho satisfactoriamente (Oberauer \& Lewandowsky, 2011).

Otra característica de los modelos formales es que los investigadores pueden comparar cuantitativamente diferentes modelos cognitivos que compiten por explicar los datos. Por consiguiente, decisiones acerca de cuál modelo ajusta mejor los datos es dada por una evaluación cuantitativa y el juicio intelectual (Lewandowsky \& Farrell, 2011). Este último aspecto es comúnmente llamado la selección de modelos (Burnham \& Anderson, 2002).

Dados el alcance y las limitaciones naturales de las teorías verbales acá se propone que los modelos 
formales son una herramienta poderosa que sirve como instrumento para una mejor integración de las teorías en psicología cognitiva y en neurociencia cognitiva. La siguiente sección describe estrategias específicas de cómo los modelos formales han contribuido con el desarrollo de ciertas teorías en neurociencia cognitiva.

Los modelos formales como puente entre el nivel cognitivoconductualy el nivel neural

Los modelos formales descomponen los datos observados en múltiples procesos cognitivos latentes; por ejemplo, el modelo de difusión (Ratcliff, 2002; Ratcliff \& McKoon, 2007) ha sido empleado para examinar los procesos cognitivos involucrados en la selección de dos alternativas simples, a saber, clasificar estímulos en términos de categorías léxicas (e.g., palabras vs no palabras), valencias (e.g., positivo vs negativo) o familiaridad (e.g., viejo vs nuevo), entre otros (Voss, Nagler \& Lerche, 2013).

El análisis tradicional de este tipo de experimentos incluye el tiempo promedio de respuesta y la proporción de decisiones correctas como variables dependientes e indicadores de la habilidad de los participantes o la diferencia en términos de dificultad entre condiciones experimentales. El modelo de difusión va más allá que el análisis estadístico tradicional al incluir la proporción de respuestas incorrectas y su tiempo promedio de respuesta como insumos para la estimación de sus parámetros. Específicamente, el modelo asume que las decisiones son tomadas con base en un proceso ruidoso de recolección de información y que la decisión se toma cuando la evidencia acumulada alcanza un umbral particular.

La Figura 2 esboza algunos supuestos del modelo de difusión y tres de sus parámetros principales. En la figura, las líneas irregulares que parten aproximadamente de la línea discontinua horizontal representan un proceso ruidoso que acumula información a través del tiempo desde un punto de inicio (i.e., el parámetro ₹ señalado por la línea horizontal discontinua) hasta el criterio de respuesta (e.g., "Correcta" o "Incorrecta") señalado por las líneas horizontales. El rango de variabilidad del parámetro z es modelado por el parámetro $s_{z}$-no mostrado en la figura.
En la Figura 2 la línea irregular que finaliza en el criterio de respuesta "Correcta" ilustra la selección de esta alternativa, la otra línea irregular ejemplifica la selección de la alternativa incorrecta. En el modelo, el parámetro drift $v$ refleja la tasa promedio de acumulación de evidencia para tomar una decisión; dicha tasa de acumulación de evidencia es determinada por la calidad de la información extraída del estímulo. Una vez que la decisión es tomada se ejecuta una respuesta, aspecto que es capturado por el parámetro $t_{0}$-en párrafos subsiguientes se describen otros componentes de este parámetro. También, el modelo permite que el parámetro $v$ varíe a través de los ensayos, dicha variabilidad es capturada por el parámetro $\eta$.

El parámetro a estima el umbral o criterio para tomar una decisión y a su vez, define un intervalo que va desde 0 hasta el valor estimado de $a$ (ver línea discontinua vertical en la Figura 1). El estimado del parámetro $a$ refleja la cautela para tomar una decisión. Cuando el valor estimado del parámetro $a$ es grande se asume que se requiere de más información para decidir entre las alternativas. Contrariamente, valores pequeños del parámetro $a$ se interpretan como que la decisión requiere de menos información. En términos empíricos, lo anterior se traduce en breves tiempos de respuesta y una menor proporción de aciertos.

Como se mencionó en el párrafo anterior el modelo de difusión incluye el parámetro $t_{0}$ el cual captura el tiempo empleado en la ejecución de la respuesta y la codificación del estímulo. El parámetro $s_{t}$ estima el rango de variabilidad de $t_{0}$ a través de los ensayos.

En resumen, la distribución de los tiempos de respuesta en el modelo de difusión se descompone en parámetros que reflejan el tiempo de decisión (i.e., $z, s_{z}$ $v, \eta$ y a) y parámetros implicados en el tiempo de $n o$ decisión (i.e., $t_{0}$ y $s$ ).

Teniendo una idea general de cómo los tiempos de respuesta son separados en parámetros que reflejan procesos cognitivos es posible establecer condiciones experimentales en las que se esperan efectos específicos sobre algunos parámetros del modelo. Por ejemplo, un estudio de Mulder et al. (2012) examinó si decisiones 


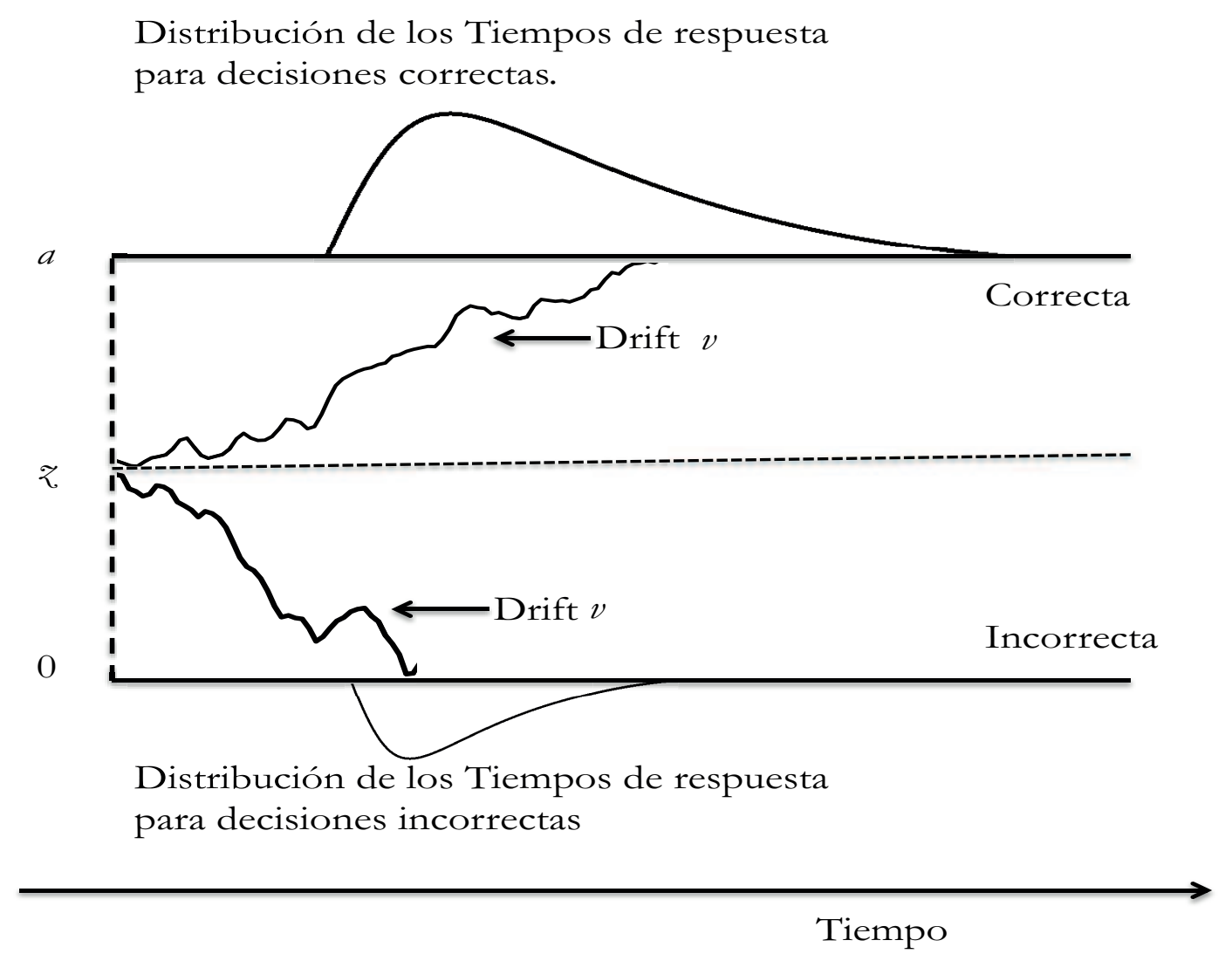

Figura 2. Representación esquemática del modelo de difusión (Ratcliff, 2002; Ratcliff y McKoon, 2007).

perceptuales pueden ser influidas por información que se presenta previamente, en concreto, se exploró si información relacionada con la probabilidad de ocurrencia de una alternativa o un refuerzo positivo (i.e., potencial pago de dinero) respecto a una de las alternativas a elegir sesgaban la decisión de los participantes.

Los investigadores propusieron que en el marco del modelo de difusión el punto de inicio para la acumulación de evidencia (i.e., parámetro $z$ ) o la tasa de acumulación de evidencia del proceso de decisión (i.e., parámetro v) podrían ser afectados por la presentación anticipada de información. Además, Mulder et al. evaluaron la posibilidad de que un sustrato neural común se encontrara relacionado con los cambios en estos parámetros.

Para estos propósitos, el estudio de Mulder et al. (2012) utilizó el paradigma de movimiento aleatorio de puntos. En este paradigma los participantes son instruidos para decidir si un subconjunto de puntos se mueve hacia la izquierda o hacia la derecha; la Figura 3 esquematiza las condiciones experimentales del estudio de Mulder et al. (2012). El Panel A muestra la secuencia de eventos para la condición probabilidad. En esta condición, primero se presenta una imagen con un punto de fijación. Luego se presenta una clave que consiste en una flecha señalando al lado izquierdo o al lado derecho. En el Panel A el signo de porcentaje en el interior de la flecha indica que existe una alta probabilidad de que los puntos se muevan a la izquierda. Posteriormente, se presenta de nuevo el punto de fijación seguido por la diapositiva que muestra puntos en movimiento, una proporción de puntos -variable a través de los ensayos- se mueve a la izquierda y el resto de los puntos se mueve aleatoriamente; durante la 
presentación de esta diapositiva los participantes deben emitir una respuesta en un periodo no mayor a 1500 ms. Los ensayos en los que la información presentada por la clave es contingente con la alternativa correcta fueron denominados como válidos, mientras que en los que la clave y la alternativa correcta no son contingentes fueron denominados ensayos no válidos. Finalmente, una diapositiva proporciona información respecto a la precisión de la respuesta la cual es indicada por la cantidad de créditos ganados en el presente ensayo. Los paneles B y C de la Figura 3 esbozan la condición experimental de refuerzo positivo ante el éxito en la tarea y la condición que no proporciona información previa (i.e., condición control), respectivamente. Los ensayos en los que no se presenta la clave fueron denominados neutros.

Con excepción de la presentación de la clave las tres condiciones experimentales fueron idénticas. Además, en tres de cuatro sesiones la actividad neuronal de los participantes fue registrada mediante fMRI.

En general, los resultados del estudio mostraron que la información presentada anticipadamente a la fase de elección tiene un efecto sobre la decisión de los participantes. Por un lado, en los ensayos válidos (i.e., cuando la clave y la alternativa presentada eran contingentes) los tiempos de respuesta eran más breves y la proporción de respuestas correctas era mayor que en ensayos inválidos o neutros. Por otro lado, los estimados del modelo de difusión indicaron que es el parámetro ₹ quien mejor explica el efecto de las condiciones experimentales sobre la decisión de los participantes. Estos hallazgos sugieren que la manipulación experimental sesga las respuestas hacia una u otra decisión más no afecta la calidad de la evidencia acumulada y con ello, la tasa de acumulación de evidencia capturada por el parámetro $v$ se mantiene igual a través de las condiciones experimentales. Además, los tiempos de respuesta al seleccionar una alternativa incorrecta fueron más prolongados para los ensayos válidos. Este hallazgo es claramente predicho por el modelo de difusión pues una vez que se presenta la clave, el punto de inicio cambia hacia alternativa

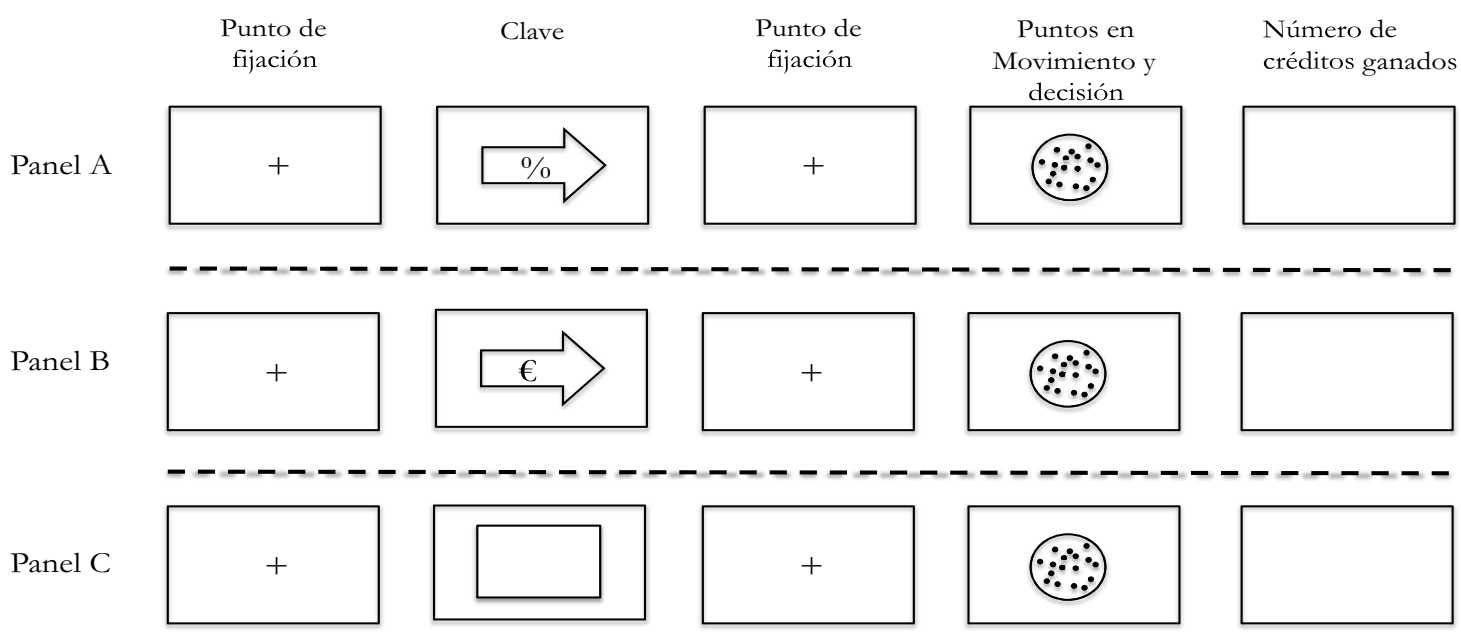

Tiempo

Figura 3. Representación esquemática de las condiciones experimentales del estudio de Mulder et al. 2012. 
que es representada por la clave y consecuentemente, el límite demarcado para la alternativa incorrecta se encuentra más lejos del estimado del parámetro \%:

Como se mencionó previamente, uno de los objetivos del estudio de Mulder et al. (2012) fue explorar la posibilidad de un sustrato neural común para el sesgo perceptual entre dos alternativas resultado de la presentación previa de información. El estudio encontró que regiones de la red frontoparietal están relacionadas con el cambio en el punto de inicio para ambos tipos de manipulaciones experimentales (Mulder et al., 2012).

A partir de este ejemplo es fácil concluir la información que añaden los modelos formales a las investigaciones de la cognición humana. Por un lado, las predicciones realizadas a partir de un modelo cognitivo formal son más precisas ya que están circunscritas a una teoría claramente especificada, lo cual a la vez permite ver los parámetros como estimados de procesos latentes. Otra ventaja de utilizar estimados de procesos latentes es que los efectos de factores extraños son minimizados, lo que conlleva a una estimación más precisa del proceso o mecanismo en cuestión (Forstmann, et al. 2011). Como se evidenció en el estudio de Mulder et al. (2012) los tiempos de respuesta y la precisión de la misma, calculados como parámetros estadísticos, ensombrecen los mecanismos cognitivos involucrados en el sesgo perceptual.

Después de todo, los modelos basados en técnicas estadísticas no son más que modelos descriptivos del objeto de estudio en cuestión, con la excepción de técnicas estadísticas que permiten la estimación de constructos latentes, por ejemplo, el modelamiento de estructuras de covarianza. Además, parámetros estimados a partir de modelos cognitivos formales permiten establecer a priori predicciones del tipo: el parámetro ₹ y/o el parámetro $v$ podrían verse afectados por la condición experimentales A mas no deberían verse afectados por condición $\mathrm{B}$, y el parámetro $t_{0}$ no debería verse afectado por ninguna de las condiciones experimentales. Este tipo predicciones hace que las teorías generadas sean mucho más poderosas en términos explicativos.
Por otro lado, el estudio de Mulder et al. (2012) ilustra cómo los parámetros derivados del modelo de difusión pueden ser utilizados como predictores de datos generados por medidas de la actividad neuronal (Forstmann, et al. 2011). Lo anterior ejemplifica la forma en que los modelos cognitivos formales orientan y fortalecen los hallazgos de estudios basados en tecnologías que proporcionan información del funcionamiento neural. Además, este estudio demuestra la sensibilidad de los parámetros latentes ante las manipulaciones experimentales y las diferencias individuales (Forstmann et al., 2011; Yechiam, Busemeyer, Stout \& Bechara, 2005)

Finalmente, es necesario recalcar que el conocimiento generado en la neurociencia cognitiva también puede ser empleado para restringir los modelos cognitivos formales, guiar su desarrollo e incluso puede ser usado para seleccionar entre modelos que compiten por explicar los datos (Forstmann et al. 2011).

\section{Recomendaciones}

En los siguientes párrafos se puntualizan algunas recomendaciones que espero sean de utilidad para todos aquellos interesados en incorporar un enfoque neurocientífico en sus líneas de investigación:

- Haga una revisión detallada de la literatura concerniente a su línea de investigación con el objetivo de conocer la forma en que se ha abordado su objeto de estudio desde las neurociencias. Esta revisión debe ir orientada a la búsqueda de resistencias que se pueden encontrar en diversas áreas de conocimiento. Evalué cada una de las posiciones encontradas y haga su mayor esfuerzo en determinar si se encuentran científicamente justificadas. Por ejemplo, si encuentra un punto de vista sólido que se opone a la incorporación de enfoques neurocientíficos es posible que su área de estudio requiera mucha investigación en el nivel cognitivoconductual para que las mediciones cerebrales sean sensibles o brinden información de su objeto de estudio. Por el contrario, si las posiciones teóricas sugieren que es un buen momento para aventurarse en las neurociencias investigue cuales tecnologías (e.g., EEG, fMRI) son las más adecuadas para sus intereses científicos y evalué 
si los hallazgos encontrados a la fecha son sólidos y consistentes con las importantes teorías cognitivas. Es importante tomar en cuenta que son las teorías cognitivas quienes deben guiar la investigación en neurociencia cognitiva. Esto le permitirá, además, valorar si las teorías se complementan o son diferentes a las desarrolladas en su ámbito de investigación (e.g., recuerde el constructo de la consolidación en neurociencias).

- Evalué si las teorías detrás de las dinámicas de su objeto de estudio han sido desarrolladas como teorías verbales o modelos cognitivos formales. Por un lado, si las teorías verbales son las que predominan en su área de investigación verifique que el diseño experimental riguroso sea una de las principales fuentes de contrastación y desarrollo de hipótesis y teorías. Si los diseños experimentales no son típicos en su área de investigación es probable que los estudios de caso, observaciones de la vida real y otras metodologías sean las más importantes en su área de interés. En dichas circunstancias sea muy cuidadoso o cuidadosa en incorporar teorías neurocientíficas y medidas cerebrales en su línea de investigación. Por otro lado, si en el ámbito de su área de estudio los modelos cognitivos formales han sido ampliamente desarrollados piense en plantear su investigación de manera que el buen diseño experimental y los modelos cognitivos formales le permitan contrastar diferentes teorías y explotar al máximo las posibilidades presentadas en la sección anterior.

Es importante señalar que estas recomendaciones están orientadas a la investigación básica y que no es propósito de esta revisión generalizarlas a la investigación aplicada, clínica o al desarrollo de tecnologías. Además, este trabajo no pretende ser exhaustivo sino que se espera que el lector lo visualice como un texto pivote para profundizar en un tópico de creciente interés. Es tal la necesidad de que las investigadoras y los investigadores se entrenen en como aventurarse en el campo de la neurociencia cognitiva que la editorial Springer tiene en prensa el libro titulado "An introduction to model-based cognitive neuroscience" Editado por Forstmann, B. U. Y Wagenmakers, E.-J.

En síntesis, este trabajo señaló resistencias y riesgos respecto a la integración de teorías entre la psicología cognitiva y neurociencias afines. Luego se señalaron las virtudes de los modelos cognitivos formales con respecto a las teorías verbales y se presentaron algunos ejemplos de cómo la incorporación de los modelos cognitivos formales facilita y mejora el desarrollo del conocimiento en la neurociencia cognitiva. En resumen, el presente trabajo sugiere que una manera adecuada - pero no la única- de reducir las asperezas entre la psicología cognitiva y las neurociencias de la cognición humana es mediante la investigación basada en modelos cognitivos formales.

\section{Referencias}

Baddeley, A. D., Thomson, N. \& Buchanan, M. (1975). Word length and the structure of shortterm memory. Journal of Verbal Learning and Verbal Behavior, 14, 575-589.

Barrouillet, P., Bernardin, S. \& Camos, V. (2004). Time constraints and resource sharing in adults' working memory spans. Journal of Experimental Psychology: General, 133, 83-100.

Barrouillet, P., Bernarding, S., Portrat, S., Vergauwe, E. \& Camos, V. (2007). Time and cognitive load in working memory. Journal of Experimental Psychology: Learning, Memory, and Cognition, 33, 570-585.

Bennett, M. R. \& Hacker, P. M. (2006). Language and cortical function: Conceptual developments. Progress in Neurobiology, 80, 20-52.

Brown, G. D. A. \& Lewandowsky, S. (2010). Forgetting in memory models: Arguments againts trace decay and consolidation failure. In S. Della Sala (Ed.), Forgetting (pp. 49-75). Hove: Psychology Press.

Brown, S. D. (2012). Common ground for behavioural and neuroimaging research. Australian Journal of Psychology, 64, 4-10.

Camos, V., Lagner, P. \& Barrouillet, P. (2009). Two maintenance mechanisms of verbal information in working memory. Journal of Memory and Language, 61, 457-469.

Colthear, M. (2012). The cognitive level of explanation. Australian Journal of Psychology, 64, 11-18. 
Corrado, G. \& Doya, K. (2007). Understanding neural coding through the model-based analysis of decision making. Journal of Neuroscience, 27, 8178-8180.

Dolan, R. J. (2008). Neuroimaging of cognition: past, present, and future. Neuro, 60, 496-502.

Farrell, S. \& Lewandowsky, S. (2010). Computational models as aids to better reasoning in psychology. Current Directions in Psychological Science, 19, 329-335.

Forstmann, B. U., Wagenmakers, E.-J., Eichele, T., Brown, S. \& Serences, J. T. (2011). Reciprocal relations between cognitive neuroscience and formal cognitive models: Opposites attract? Trends in Cognitive Sciences, 15, 272-279.

Gallistel, C. R. (1999). Themes of thought and thinking. In R. J. Sternberg (Ed.), The Nature of Cognition. Science, 285, 842-843.

Kieras, D. E., Meyer, D. E., Mueller, S. \& Seymour, T. (1999). Insights into working memory from the perspective of the EPIC architecture for modelling skilled perceptualmotor and cognitive human performance. In A. Miyake and P. Shah (Eds.), Models of working memory: Mechanisms of active maintenance and control (pp. 183-223). New York, NY: Cambridge University Press.

Kihlstrom,J. F. (2010). Social neuroscience: The footprints of Phineas Gage. Social Cognition, 28, 757-783.

Kim, J-J., Kim, M. S., Lee, J. S., Lee, D. S., Lee, M. C., \& Kwon, J. S. (2002). Dissociation of working memory processing associated with native and second languages: PET investigation. NeuroImage, 15, 879-891.

Lewandowsky, S. (1993). The rewards and hazards of computer simulations. Psychological Science, 4, 236-243.

Lewandowsky, S. \& Farrell, S. (2011). Computational Modeling in Cognition: Principles and Practice. Thousand Oaks, CA: Sage.

Lewandowsky, S., Ecker, U. K. H., Farrell, S. \& Brown, G. D. A. (2012). Models of cognition and constraints from neuroscience: A case study involving consolidation. Australian Journal of Psychology, 64, 37-45.

Mercier, H. \& Sperber, D. (2011). Why do humans reason? Arguments for an argumentative theory. Behavioral and Brain Sciences, 34, 57-111.

Mulder, M. J., Wagenmakers, E.-J., Ratcliff, R., Boekel, W., \& Forstmann, B. U. (2012). Bias in the brain: A diffusion model analysis of prior probability and potential payoff. The Journal of Neuroscience, 32, 2335-2343.

Naughtin, C. K., Mattingley, J. B. \& Dux, P. E. (2014). Distributed and overlapping neural substrates for object individuation and identification in visual short-term memory. Cerebral Cortex. Advance online publication. doi:10.1093/cercor/bhu212

O'Doherty, J. P., Hampton, A. \& Kim, H. (2007) Model-based fMRI and its application to reward learing and decision making. Annals of the New York Academy of Sciences, 1104, 35-53.

Oberauer, K. \& Kliegl, R. (2006). A formal model of capacity limits in working memory. Journal of Memory and Language, 55, 601-626.

Oberauer, K. \& Lewandowsky, S. (2011). Modeling working memory: A computational implementation of the Time-Based Resource-Sharing theory. Psychonomic Bulletin \& Review, 18, 10-45.

Oberauer, K. Lewandowsky, S., Farrell, S., Jarrod, C. \& Greaves, M. (2012). Modeling working memory: An interference model of complex span. Psychonomic Bulletin \& Review, 19, 779-819.

Pages, M. P. A. \& Norris, D. (1998). The primacy model: A new model of immediate serial recall. Psychological Review, 105, 761-781.

Peigneux P., Schmitz R., \& Urbain, C. (2010). Sleep and Forgetting. In S. Della Sala (Ed.), Forgetting (pp. 165184). Hove: Psychology Press.

Ratcliff, R. (2002). A diffusion model account of reaction time and accuracy in a two choice brightness discrimination task: Fitting real data and failing to fit fake but plausible data. Psychonomic Bulletin and Review, 9, 278-291.

Ratcliff, R. \& McKoon, G. (2007). The diffusion decision model: Theory and data for two-choice decision task. Neural Computation, 20, 873-922. 
Sauseng, P., Klimesch, W., Heise, K. F., Gruber, W. R., Holz, E., Karim, A. A.,... Hummel, F. C. (2009). Brain oscillatory substrates of visual short-term memory capacity. Current Biology, 19, 1846-1852.

Sternberg, R. J. \& Sternberg, K. (2011). Cognitive Psychology. Cengage Learning: Belmont California.

Vogel, E., McCollough, A. W. \& Machizawa, M. G. (2005). Neural measures reveal individual diferences in controlling access to working memory. Nature, $438,500-503$.

Voss, A., Nagler, M. \& Lerche, V. (2013). Diffusion models in experimental psychology: a practical introducction. Experimental Psycbology, 60, 385-402.
Weisberg, D. S., Keil, F. C., Goodstein, J., Rawson, E. \& Gray, J. R. (2008). The seductive allure of neuroscience explanations. Journal of Cognitive Neuroscience, 20, 470-477.

Wixted, J. T. (2004). The psychology and neuroscience of forgetting. Annual Review of Psychology, 55, 235-269.

Wixted, J. T. (2010). The role of retroactive interference and consolidation in everyday forgetting. In S. Della Sala (Ed.), Forgetting (pp. 285-312). Hove: Psychology Press.

Yechiam, E., Busemeyer, J. R., Stout, J. C. \& Bechara, A. (2005). Using cognitive models to map relations between neuropsychological disorders and human decision-making déficits. Psychological Science, 16, 973-978.

Recibido: 05 de mayo 2014 Aceptado: 20 de agosto 2014 